MEN ${ }^{\circledast}$; site of administration VISIne ${ }^{\circledast}$ (vision), OPTIcure ${ }^{\circledast}$, PhotoDERM ${ }^{\circledast}$; dosage form as Enemacort (ENEMA CORTisone), Folicap ${ }^{\oplus}$ (FOLIc acid CAPsules); dose regimen and duration Cefobid $^{\circledast}$ (CEPHalosporin BID-twice daily), Lasix it's effect LAst for SIX hours, Slow $K^{\circledast}$ is a SLOW release potassium $(K)$, Novorapid $^{\boxplus}$ reflects short and Rapid acting insulin; drug history or story Nystatin ${ }^{\circledast}$ was named after the New York(NY) STATE, while warfarin ${ }^{\circledR}$ is an acronym for Wisconsin Alumni Research Foundation(W.A.R.F). Trade names affected by brand name as Glimaryl $l^{\circledast}$ affected by Amaryl $^{\circledR}$ while Marivanil $^{\circledR}$ affected by Marivan ${ }^{\boxplus}$.

Conclusions The research highlights 12 observed patterns used in pharmaceutical trade naming. Implementation of these methods will help the students, pharmacists and health care providers to become more aware of the message that the drug trade name delivers.

\section{HOW MUCH DO GENERAL PRACTITIONERS KNOW ABOUT THE ABSOLUTE VALUE AND POSSIBLE HARMS OF TREATMENTS FOR COMMON LONG-TERM CONDITIONS? A QUESTIONNAIRE SURVEY}

Julian Treadwell. University of Oxford, Oxford, UK

\subsection{6/bmjebm-2019-EBMLive.63}

Objectives In Britain, GPs are responsible for prescribing multiple long-term treatments to their patients. To support shared clinical decision making, understanding of the absolute benefits and harms of individual treatments is needed. International evidence shows that doctors' knowledge of absolute treatment effects is poor, but this has not been researched among British GPs.

Aim To assess and describe the level and range of the quantitative understanding of the benefits and harms of treatments for common long-term conditions among British GPs.

Method An online survey distributed to GPs in Britain over two months in 2018. Participants were asked to estimate the percentage absolute risk reduction or risk increase conferred by 13 interventions across 10 long term conditions on 17 important outcomes. Responses were collated and presented graphically for each clinical question and analyses performed to estimate the proportion of correct responses.

Results 443 respondents, broadly representative of the British GP population, were included in the analysis. The majority of respondents demonstrated poor knowledge of the absolute benefits and harms of treatments with inaccuracies common and wide ranging. Per question, only $3.2-28.4 \%$ of responses were correct allowing for $+/-1 \%$ margin in ARR estimates and $10.4-55.6 \%$ allowing a $+/-3 \%$ margin. $65 \%$ of GPs self-reported low to very low confidence in their knowledge.

Conclusions GPs' knowledge of the absolute benefits and harms of treatments is poor, with inaccuracies of a magnitude likely to significantly affect clinical decision making and impede meaningful conversations with patients regarding treatment choices.

This represents a barrier to the practice of EBM as it is intended. The causes are complex and lie within the system of evidence dissemination, implementation and performance management of practitioners. These will be discussed along with potential solutions.

\section{UNDERSTANDING BIAS, CONFLICTS OF INTEREST AND RESEARCHER ALLEGIANCE IN SYSTEMATIC REVIEWS}

Lesley Uttley. University of Sheffield, Sheffield, UK

10.1136/bmjebm-2019-EBMLive.64

Objectives Systematic reviews and meta-analyses are the foundation of modern evidence-based medicine and their use is becoming more prolific than randomised trials. However, systematic reviews are receiving increasing criticism for contributing to research waste, varying massively in their reporting or methodological quality, being misleading and serving conflicted interests. ${ }^{1}$ Systematic reviews are upheld as being objective, dispassionate scientific processes but they can bias the evidence base. Influential decisions are made by people at all stages of a review including question setting, searching, study selection, interpretation, and making recommendations for research or practice. We know that biased research is prevalent in primary research and it is becoming increasingly apparent that questionable research practices also affect systematic reviews.

- Improve the systematic use of existing evidence by exploring and highlighting biases and poor conduct in systematic reviews

- Emphasise under-recognised biases or influences that can affect systematic reviews and disseminate them in a clear and accessible way to relevant audiences including health researchers, policy makers and journal editors

Method Initiatives for assessing methodological rigour and reporting such as Cochrane or PRISMA do not discuss errors, misconduct or bias which are attributable to the review team, and such influences can affect the reliability and validity of evidence syntheses.

Standard conflicts of interest (CoI) statements focus on narrow commercial interests and are inadequate to address potentially hidden agendas in systematic review teams. For example, CoI are frequently not declared as such by review authors who are psychological therapists when reviewing their own psychotherapy (researcher allegiance). Reviews with a high number of authors affiliated to the intervention are more likely to have positive conclusions and more likely to be lower quality reviews (confirmation bias). At least a quarter of investigators in biomedical research have industry affiliations, and a significant relationship exists between industry sponsorship and pro-industry conclusions (sponsorship bias).

Results Stakeholder participation in reviews, whilst being potentially beneficial, can also be problematic to manage as involvement of content area experts can make it more difficult to perform an unbiased review (one-sided reference bias). The opportunity to assess why the review authors were motivated to assess the evidence base is too limited if relying on information such as funding source and the expression of pecuniary conflicts.

Currently consumers of systematic reviews cannot rely on journal publication declarations to know whether those conducting a review have vested interests or are appropriately skilled. Policy decisions which ultimately affect patient care are influenced by systematic reviews therefore the integrity of their conduct requires more scrutiny.

Potential solutions:

- Empirical research to scrutinise less obvious CoI and examine the impact on published systematic reviews conclusions 
- A dedicated website to clearly explain biases that systematic reviews are prone to

- Guidelines to help peer-reviewers, journal editors and the public understand appropriate systematic review team composition and assess potential for bias or poor conduct.

\section{REFERENCE}

1. Ioannidis J. The mass production of redundant, misleading, and conflicted systematic reviews and meta-analyses. The Milbank Quarterly. 2016;94(3):485-514.

\section{ESTIMATING THE EVIDENCE GAP IN NETWORK META-ANALYSIS}

Ghazaleh Aali, Clive E Adams, Farhad Shokraneh*. Cochrane Schizophrenia Group, Institute of Mental Health, Division of Psychiatry and Applied Psychology, School of Medicine, University of Nottingham, Nottingham, UK

10.1136/bmjebm-2019-EBMLive.65

Objectives Network meta-analysis (NMA) provides plots of interventions networks per outcome. Such plots also demonstrate all possible pairwise comparisons in a network. This paper discusses the gap between evidence expected from a given network and observed evidence from trials by use of a simple formula.

Method Published network meta-analyses in BMJ, JAMA, Lancet and New England Journal of Medicine were collected (2003-2019). The potential number of comparisons per network plot was calculated using $\left(\mathrm{n}^{*}(\mathrm{n}-1)\right) / 2^{1}$ and the number of direct comparisons from trials was deducted to obtain the 'evidence gap' per network plot. We also compared evidence gap in the networks with low or high number of intervention nodes. All steps were conducted by GA, repeated by FS and supervised by CEA.

Results We excluded four NMAs because of mixing study designs but were able to include 41 NMAs of randomised controlled trials (RCTs). We identified 77 network plots from NMAs. The plots consisted of between 2 and 31 intervention nodes. Only four plots were complete and based on only direct comparisons. The evidence gap was between 0.06 and 0.88 for the remainder - between $10 \%$ and $90 \%$ of the comparisons in the network have not yet been reported in RCTs. There is a positive and strong correlation between the number of interventions and the number of indirect comparisons $\left(\mathrm{R}^{2}\right.$ $=0.894$ ) highlighting the considerable gap in the certainty of body of evidence as presented in NMAs. The evidence gap is filled statistically without real-world evidence from trials and the results of NMA are particularly problematic when there are many interventions in the plot.

Conclusions Researchers who undertake NMAs should report network plots and a list of missing comparisons from the trials. They should also report the evidence gap to emphasise the proportion of the NMA which is based on data derived from real world experiments and the proportion from statistic-based inference. The findings of our research call for an update of the PRISMA for Network Meta-Analyses reporting guideline.

\section{REFERENCE}

1. Shokraneh F, Adams CE. A simple formula for enumerating comparisons in trials and network meta-analysis. F1000Research. 2019 Jan 9; 8:38. DOI 10.12688/ f1000research. 17352.1

\section{OUR DOCTORS NEED HELP! - 'EVIDENCE BASED MEDICINE DOCTORS INFORMATION CENTERS' COULD BE THE KEY TO FACILITATE DAILY PRACTICE}

Theresa Schmalfuß. The Cochrane Collaboration Austria, Krems (Vienna Area), Austria

\subsection{6/bmjebm-2019-EBMLive.66}

Our doctors need help! - 'Evidence Based Medicine Doctors Information Centers' could be the key to facilitate daily practice Every month 150.000 articles are published in 20.000 different medical journals. In order to stay up-to-date with constantly increasing research one needs to read 500 Studies each week. That is $1,5 \mathrm{~m}$ of paper every week. As a comparison: in 1980 it was it merely 1 study per week one had to read. As medical professional it can thus be very challenging and time consuming to find good and solid evidence in a myriad of research of today's society that is pressured to constantly publish new data and information. Even guidelines can be written incomprehensibly or might even be contradicting themselves. In order to critically question studies, some practice and additional training are needed, which are often neglected in medical education. Many doctors thus lack essential skills to select, understand and critically appraise information that is being presented to them. We need more tools for clinical practice that facilitate decision making in evidence-based medicine. The 'evidence-based medicine information center for doctors' is a great example of how this can be achieved. It is a project, originally initiated by the Cochrane Collaboration Austria, that answers clinical questions from everyday hospital life in the form of rapid reviews. The great thing about rapid reviews is that evidence is synthesized more quickly than in a systematic review, since certain methodological aspects are abbreviated, and all the important knowledge is compressed in a short and understandable matter. For each question the first step is a systematic literature search in several databases. Two research assistants then examine a large number of international studies to see whether they fit the question. After abstract screening all articles that are shortlisted are examined again and the entire study is checked for its quality, i.e. whether the methodological implementation meets internationally recognized standards. Finally, an assessment of how much confidence the Information Centre has in the results of the selected studies is included in each Rapid Review, which gives the doctors an explanation of how strong and trustworthy this evidence is. This tool, specifically designed for doctors, gives guidance in clinical decision making and answers, 'real life' and relevant questions that arise when good evidence cannot be found, is not understood or guidelines are simply unclear. Of course, one could argue that training could be a more efficient way to equip doctors with the skills necessary to assess evidence. But let's be realistic - who has the time? This tool should be implemented in every country to give our doctors the possibility to come into contact with those that have been trained to synthesize and assess evidence effectively. The clinical decisions our doctors make directly affect the health of our patients, so it should be our main priority to find and implement a solution that supports our medical professionals to make an informed and fair decision. If it works in Austria, why wouldn't it in other countries? 